\title{
Um estudo sobre o responder relacional de comparação com base na aceitação de substâncias psicoativas utilizando o IRAP*
}

\author{
A study on the relational response of comparison based on the \\ acceptance of psychoactive substances using the IRAP \\ Un estudio sobre respuesta relacional de la comparación basada en la \\ aceptación de sustancias psicoactivas utilizando el IRAP
}

\author{
César Silva Rodrigues Oliveira', João Henrique de Almeida ${ }^{2}$, Renato Bortoloti', Thais Porlan de Oliveira', \\ Edson Massayuki Huziwara ${ }^{1}$
}

\begin{abstract}
[1] Universidade Federal de Minas Gerais [2] Universidade Estadual de Londrina | Título abreviado: Responder relacional de comparação entre substâncias psicoativas I Endereço para correspondência: Edson Huziwara. Rua Araripe, 10, apto 1001. Belo Horizonte, MG. CEP $31015-26$ | Email: huziwara.edson@gmail. com I doi: 10.18761/PAC.2021.v12.RFT.06
\end{abstract}

\begin{abstract}
Resumo: O presente estudo objetivou avaliar, por meio do Implicit Relational Assessment Procedure (IRAP), a existência de um responder relacional de comparação com base na aceitação de substâncias psicoativas. Esperava-se que, mesmo pessoas que nunca consumiram tais substâncias, pudessem desenvolver uma ordem de aceitação semelhante à ordem de consumo dessas drogas no Brasil (i.e., álcool mais aceitável que o tabaco, tabaco mais aceitável que a maconha e maconha mais aceitável que a cocaína). Estudantes universitários com baixos níveis de consumo de álcool e sem história prévia de uso de tabaco, maconha e cocaína participaram do estudo. Os participantes preencheram quatro escalas Likert para aferir a aceitação de cada substância psicoativa e responderam a um IRAP programado para avaliar o responder relacional de comparação entre as quatro substâncias psicoativas mencionadas anteriormente. Nossos resultados não confirmaram a hipótese de que a ordem de aceitação das substâncias psicoativas seguiria a ordem de prevalência de uso destas substâncias. Em uma análise post hoc, somente os resultados do IRAP serviram para diferenciar participantes com e sem experiência prévia com álcool. São realizadas considerações a respeito da relação entre atitudes específicas sobre substâncias psicoativas e seu uso, bem como sobre a programação dos estímulos no IRAP e suas limitações para avaliar o responder relacional de comparação.
\end{abstract}

Palavras-chave: Implicit Relational Assessment Procedure; responder relacional de comparação; substâncias psicoativas; aceitação; prevalência

\footnotetext{
*Esta pesquisa contou com o apoio do Instituto Nacional de Ciência e Tecnologia em Comportamento, Cognição e Ensino (INCT - ECCE), financiado pela Fundação de Amparo à Pesquisa do Estado de São Paulo (FAPESP, processo no 2014/50909-8), pela Coordenação de Aperfeiçoamento de Pessoal de Nível Superior (CAPES, processo nº 88887.136407/2017-00), e pelo Conselho Nacional de Desenvolvimento Científico e Tecnológico (CNPq, processo ${ }^{\circ}$ 465686/2014-1).
} 


\begin{abstract}
The present study aimed to evaluate, through the Implicit Relational Assessment Procedure (IRAP), the existence of a comparative relational response based on the acceptance of psychoactive substances. It was possible to argue that even people who have never consumed such substances would be able to develop an order of acceptance similar to the order of consumption of these drugs in Brazil (i.e., alcohol more acceptable than tobacco; tobacco more acceptable than marijuana; and marijuana more acceptable than cocaine). Undergraduate students with low levels of alcohol consumption and with no previous history of tobacco, marijuana, and cocaine use participated in the study. Participants completed four Likert scales to assess the acceptance of each psychoactive substance and responded to an IRAP programmed to assess the relational response of comparison between the four psychoactive substances previously mentioned. Our findings did not confirm the hypothesis that the order of acceptance of psychoactive substances would follow the order of prevalence of use of these substances. In a post hoc analysis, only the data from IRAP served to differentiate participants with and without previous experience with alcohol. Considerations are made regarding the relationship between specific attitudes about psychoactive substances and their use, as well as about the programming of stimuli in IRAP and their limitations for assessing the relational response of comparison.
\end{abstract}

Keywords: Implicit Relational Assessment Procedure; relational responding of comparison; psychoactive substances; acceptance; prevalence

Resumen: El presente estudio tuvo como objetivo evaluar, a través del Procedimiento de Evaluación Relacional Implícita (IRAP), la existencia de una respuesta relacional comparativa basada en la aceptación de sustancias psicoactivas. Se esperaba que incluso las personas que nunca habían consumido tales sustancias pudieran desarrollar una orden de aceptación similar al orden de consumo de estas drogas en Brasil (es decir, alcohol más aceptable que el tabaco, tabaco más aceptable que la marihuana y marihuana más aceptable que la cocaína). En el estudio participaron estudiantes universitarios con bajo consumo de alcohol y sin antecedentes de consumo de tabaco, marihuana y cocaína. Los participantes completaron cuatro escalas Likert para evaluar la aceptación de cada sustancia psicoactiva y respondieron a un IRAP programado para evaluar la respuesta relacional de comparación entre las cuatro sustancias psicoactivas mencionadas anteriormente. Nuestros resultados no confirmaron la hipótesis de que el orden de aceptación de sustancias psicoactivas seguiría el orden de prevalencia del uso de estas sustancias. En un análisis post hoc, solo los resultados del IRAP sirvieron para diferenciar a los participantes con y sin experiencia previa con alcohol. Se hacen consideraciones sobre la relación entre actitudes específicas sobre sustancias psicoactivas y su uso, así como sobre la programación de estímulos en IRAP y sus limitaciones para evaluar la respuesta relacional de comparación.

Palabras clave: Procedimiento de Evaluación Relacional Implícita; respuesta de comparación relacional; sustancias psicoactivas; aceptación; predomínio 
Ao longo dos últimos anos, muitos experimentos utilizaram o Implicit Relational Assessment Procedure (IRAP; Barnes-Holmes, Barnes-Holmes et al., 2010) para investigar redes de relações arbitrárias entre estímulos estabelecidas por nossas histórias particulares de reforçamento (e.g., Cagney et al., 2017; Carpenter et al., 2012; Drake et al., 2015; Farrell \& McHugh, 2017; Hussey et al., 2016; Leech et al., 2016; Parling et al., 2012; Remue et al., 2013). Em linhas gerais, o IRAP pode ser descrito como uma tarefa computadorizada em que são apresentados dois estímulos de forma simultânea, um deles denominado "rótulo" e o outro "alvo". Diante dessas combinações de estímulos, os participantes são requisitados a escolher de forma rápida e acurada uma entre duas opções de resposta que especificam uma relação particular entre rótulo e alvo. Importante salientar que a escolha a ser emitida é definida pelo experimentador, podendo ser consistente com a história de aprendizagem do indivíduo em alguns blocos de tentativas e inconsistentes em outros. De acordo com as premissas teóricas que embasam o IRAP (Barnes-Holmes et al., 2010; Dawson et al., 2009; para revisão ver Hughes et al., 2011), espera-se que os indivíduos respondam mais rapidamente nas tentativas cujas respostas forem consistentes com sua história anterior de aprendizagem e mais lentamente nas tentativas cujas respostas forem inconsistentes, resultado conhecido como 'efeito IRAP'.

No experimento conduzido por Barnes-Holmes et al. (2008), por exemplo, dois grupos de participantes foram expostos a dois procedimentos IRAP distintos. Para o primeiro grupo, cada tentativa apresentava ou a palavra "AGRADÁVEL" ou a palavra "DESAGRADÁVEL" como rótulo. De forma simultânea, uma palavra valorada positivamente (e.g., "saúde", "paz", "alegria”) ou negativamente (e.g., "doença", "guerra", "tristeza") era apresentada como alvo. Os participantes tinham que escolher entre as opções de resposta "similar" ou "oposto". Nos blocos consistentes, os participantes eram instruídos a responder de maneira a refletir as contingências verbais possivelmente predominantes na comunidade, ou seja, escolher a opção "similar" para "agradável-positivo" e "desagradável-negativo" e a opção "oposto" para "agradável-negativo" e "desagradável-positivo". Nos blocos inconsistentes, os participantes eram instruídos a responder de maneira inversa, ou seja, "oposto" para "agradável-positivo" e "desagradável-negativo" e "similar" para "agradável-negativo" e "desagradável-positivo".

Para o segundo grupo de participantes, os estímulos alvo e as opções de respostas eram as mesmas utilizadas para o Grupo 1. A única diferença estava relacionada aos rótulos utilizados. Ao invés de "AGRADÁVEL" e "DESAGRADÁVEL", eram utilizadas as trigramas "CUG" e "ZID". Os resultados indicaram que apenas os participantes submetidos à tarefa com estímulos com significado pré-experimental (i.e., "AGRADÁVEL" e "DESAGRADÁVEL") tiveram menor latência média de resposta nos blocos consistentes que nos inconsistentes, enquanto os participantes que executaram a tarefa com as trigramas tiveram latência média de resposta indiferenciada entre os blocos. Em outras palavras, a diferença entre as latências médias dos blocos consistentes e inconsistentes foi obtida apenas nas situações em que estímulos com significado pré-experimental foram utilizados como rótulo, confirmando a adequação do IRAP como medida de avaliação de relações condicionais estabelecidas na história de aprendizagem dos participantes.

Apesar de as tarefas IRAP serem tipicamente formatadas dentro dos parâmetros descritos anteriormente, o número de relações avaliadas pode variar, como demonstrado no estudo de Power et al. (2009). Neste experimento, um número maior de relações foi apresentado para investigar a existência de uma ordem relacional entre os pares de estímulos avaliados. Mais especificamente, o IRAP empregou pares de palavras como alvos e expressões de comparação como rótulos no intuito de investigar se a preferência relativa por outros grupos étnicos seria ordenada a partir da similaridade social percebida em relação ao seu próprio grupo. Por exemplo, considerando os irlandeses como o grupo de referência, a ordem de preferência étnica modulada pela similaridade social percebida seria: irlandeses-escoceses-americanos-africanos. Tendo 16 cidadãos irlandeses como participantes, os estímulos rótulos nesse procedimento eram as expressões "mais simpático" e "menos simpático", enquanto os seis pares de estímulos alvos eram irlandeses-escoceses, escoceses-americanos, americanos-africanos, e suas contrapartidas, escoceses-irlandeses, americanos-esco- 
ceses e africanos-americanos. Em cada tentativa, o participante era solicitado a escolher um de dois termos relacionais, "verdadeiro" ou "falso". Assim sendo, cada tentativa apresentava uma relação de comparação entre duas etnias. Mais especificamente, a apresentação do rótulo "mais simpático" e do alvo irlandeses-escoceses deveria ser entendida como a afirmação de que irlandeses são mais simpáticos do que escoceses. Nesse exemplo, os participantes deveriam escolher a opção "verdadeiro" nos blocos consistentes e a opção "falso" nos blocos inconsistentes. A análise das latências médias para a emissão da resposta mostrou que os participantes responderam significativamente mais rápido nos blocos consistentes que nos blocos inconsistentes, sugerindo a existência de um viés que confirmava a ordem de preferência étnica modulada pela similaridade social percebida, proposta por Power et al (2009). Por outro lado, neste mesmo experimento, uma medida explícita elaborada para aferir a preferência étnica encontrou uma ordem distinta e provavelmente modulada por uma adequação das respostas àquilo que seria socialmente desejável.

De forma semelhante à questão da preferência étnica, outras temáticas poderiam ser exploradas em experimentos que fossem capazes de verificar a existência de ordenações relacionais estabelecidas a partir de histórias de aprendizagem. Porém, há uma quantidade muito limitada experimentos que investigaram a formação e existência de ordens relacionais a partir do IRAP. Por exemplo, é possível supor que indivíduos adultos sem experiência prévia com o uso de substâncias psicoativas sejam mais propensos a relacioná-las com atributos avaliativos negativos. Além disso, também é possível supor que as relações estabelecidas entre diferentes substâncias psicoativas e os referidos atributos negativos possam apresentar algum grau de ordenação, sendo algumas substâncias consideradas mais perigosas ou prejudiciais do que outras.

Interessante notar ainda que essa ordem hierárquica não precisa ser, necessariamente, relacionada com o grau efetivo de malefícios causados por determinada substância, mas pode ser controlada por contingências históricas verbais do contexto cultural preponderante. Nutt et al. (2010), por exemplo, estabeleceram uma classificação de risco de 20 substâncias psicoativas segundo 16 critérios, nove deles relacionados a danos produzidos nos próprios indivíduos e outros sete relacionados a danos causados em outras pessoas. De acordo com os autores, o álcool foi considerado a droga mais perigosa dentre as 20 substâncias psicoativas estudadas (e.g., heroína, cocaína, metanfetamina, tabaco, maconha). Neste sentido, é possível dizer que o caráter legal da substância não corresponde a seus riscos potenciais, bem como suas avaliações podem não corresponder ao grau efetivo de malefícios causados. É bem provável que a legalidade da substância seja um aspecto importante para a formação de atitudes relacionadas a ela, seja pela estigmatização do uso, pelas consequências compartilhadas ao exercício de atividades ditas "ilegais", bem como pelo efeito simbólico geral da proibição (MacCoun, 1993). Interessante salientar também que os níveis de desaprovação relacionados a uma determinada substância psicoativa são tidos como um fator de proteção para o seu uso futuro (Bachman et al., 1990), ou seja, quanto maior o nível de desaprovação de uma substância, menor seria a probabilidade de uma pessoa fazer uso dela ao longo da vida.

O debate sobre a legalização da maconha no Brasil e no mundo tem ganhado considerável destaque nos últimos anos (Kiepper \& Esher, 2014; Wilkinson et al., 2016). Simultaneamente, políticas de regulação e controle sobre outras substâncias psicoativas também estão na agenda de muitos governos, como no caso das políticas sobre a comercialização e consumo do tabaco (Laranjeira, 2010). Sabe-se que agências de controle como o governo, a mídia, a religião, a escola, a família, entre outras, são responsáveis pelo estabelecimento de contingências importantes para a formação e modificação do universo simbólico compartilhado em uma cultura (de Rose \& Bortoloti, 2007; Mizael et al., 2016; Skinner, 1953). Neste sentido, destaca-se a importância dos controles regulatórios sobre as diferentes substâncias psicoativas, bem como da atuação das agências de controle no sentido de estabelecer diferentes relações condicionais entre cada tipo de substância e diferentes níveis de atributos negativos, principalmente em indivíduos que não possuem experiência direta com as tais substâncias.

Assim sendo, a partir do tratamento diferenciado dado a cada tipo de substância, parece possível supor a existência de uma ordem em termos 
de aceitação do consumo de diferentes substâncias psicoativas, sejam elas lícitas ou ilícitas. Em um nível macroscópico, estas contingências podem ser responsáveis por determinar, ao menos em parte, a prevalência de uso de substâncias observada em uma dada sociedade. No Brasil, por exemplo, as drogas legais de maior livre acesso são o álcool e o tabaco. De acordo com os dados do II Levantamento Nacional de Álcool e Drogas (LENAD), que perguntava sobre o consumo de substâncias lícitas e ilícitas ocorrida nos últimos 12 meses que antecederam a resposta ao questionário, $50 \%$ da população adulta brasileira relatou ter consumido álcool, sendo que, dentre a população adulta não abstêmia, 53\% relatou que fez uso da substância ao menos uma vez na semana. Em relação ao tabaco, $16,9 \%$ da população adulta foi considerada fumante. Sobre drogas ilícitas, os dados revelam que maconha e cocaína foram as substâncias mais consumidas, cujas prevalências de uso corresponderam a $2,5 \%$ e $1,7 \%$, respectivamente (Laranjeira et al., 2012). Assim sendo, de acordo com a prevalência do uso dessas substâncias, é possível supor que a relação de aceitação das drogas lícitas e ilícitas mais utilizadas no contexto brasileiro deveria compreender a ordem em que álcool é mais aceitável que tabaco, tabaco mais aceitável que maconha e maconha mais aceitável que cocaína.

Com a finalidade de avaliar a existência dessa ordem relacional na aceitação do uso de substâncias psicoativas, optou-se pelo emprego do IRAP. Importante salientar que esse instrumento foi utilizado em estudos prévios para investigar o preconceito com relação ao usuário de substâncias psicoativas (Drake et al., 2018; Levin et al., 2010) e, portanto, sua utilização nesse contexto nos pareceu promissora. Este estudo foi delineado para investigar se indivíduos com ausência de história de uso de substâncias sobre as quais existe um maior controle regulatório governamental (i.e., tabaco, maconha e cocaína) confirmariam um viés em relacionar a ordem de aceitação com sua prevalência de consumo no contexto brasileiro. Além disso, os resultados obtidos no IRAP foram também comparados com resultados obtidos por meio de uma medida explícita da aceitação do uso dessas mesmas substâncias psicoativas.

\section{Método}

\section{Participantes}

A amostra inicial deste experimento contava com 66 estudantes universitários. Contudo, 32 deles tiveram sua participação interrompida porque não atingiram os critérios de tempo e acurácia exigidos durante os blocos de treino do IRAP. Além disso, outros dois participantes foram não tiveram seus dados incluídos na análise, pois não mantiveram os níveis de acurácia exigidos nos blocos de teste do IRAP. Portanto, foram analisados os dados de 32 estudantes universitários (17 do sexo masculino), com idades entre 18 e 25 anos $(\mathrm{M}=19,94$; DP $=2,08)$, os quais atingiram os critérios de tempo e acurácia durante os blocos de treino e mantiveram esses critérios durante os blocos de teste do IRAP.

Para caracterização da amostra, os participantes preencheram um instrumento de triagem sobre o uso de substâncias psicoativas denominado Alcohol, Smoking and Substance Involvement Screening (ASSIST), desenvolvido sob a coordenação da Organização Mundial de Saúde (OMS) e validado no Brasil por Henrique et al. (2004). A respeito do uso de álcool, 71,9\% dos participantes declararam já ter feito uso desta substância ao longo da vida. Dentre eles, $34,8 \%$ não haviam feito uso nos últimos três meses, $39,1 \%$ beberam de uma a duas vezes nos últimos três meses e $26,1 \%$ declararam ter bebido mensalmente nos último três meses. Todos eles declararam nunca ter feito uso de tabaco, maconha ou cocaína.

Os participantes leram e assinaram um Termo de Consentimento Livre e Esclarecido (TCLE) antes do início do experimento e não receberam qualquer contrapartida financeira para a participação do estudo. Os procedimentos utilizados no presente experimento foram aprovados pelo Comitê de Ética em Pesquisa com seres humanos (processo número CAAE: 2.596.691).

\section{Situação e equipamentos}

As atividades foram realizadas em uma sala de $28 \mathrm{~m}^{2}$ em condições adequadas para evitar interrupções desnecessárias ou ruídos estranhos à realização do experimento. Foi utilizado o programa GO-IRAP instalado em um computador desktop e 
monitor de 15 polegadas. O software foi empregado para apresentar os estímulos, liberar as consequências programadas e gravar as respostas emitidas pelos participantes.

\section{Medida explícita}

A avaliação do grau de aceitação de quatro substâncias psicoativas (i.e., álcool, tabaco, maconha e cocaína) foi medida explicitamente por meio de quatro escalas Likert. Cada escala apresentava o nome de uma substância na parte superior da folha e, logo abaixo, uma sequência horizontal de 13 espaços. As extremidades de cada escala eram ancoradas com as expressões 'extremamente aceitável' e 'extremamente inaceitável'. O participante era requisitado a assinalar o espaço que melhor refletisse a sua opinião quanto ao grau aceitação da substância especificada no topo da folha. Assim, se o participante considerasse álcool como extremamente aceitável, deveria marcar o espaço mais próximo à expressão "extremamente aceitável"; por outro lado, se o considerasse como extremamente inaceitável, deveria marcar o espaço mais próximo da expressão "extremamente inaceitável". Finalmente, o participante deveria marcar o espaço equidistante de ambas as extremidades caso considerasse a substância igualmente aceitável e inaceitável. A ordem de avaliação das substâncias diferiu entre os participantes. De acordo com o ponto escolhido ao longo deste continuum, era atribuído um valor que variava de - 6 (extremamente inaceitável) a +6 (extremamente aceitável).

\section{Implicit Relational Assessment Procedure (IRAP)}

Cada tentativa do IRAP apresentava um de dois estímulos rótulo: "mais aceitável que" ou "menos aceitável que". Simultaneamente, era apresentado um entre seis alvos: álcool-tabaco, tabaco-maconha, maconha-cocaína, e as contrapartidas, tabaco-álcool, maconha-tabaco e cocaína-maconha. De forma similar ao ocorrido no experimento de Power et al. (2009), uma tentativa que apresentasse o rótulo "mais aceitável que" e o alvo álcool-tabaco deveria ser entendida como a afirmação: álcool é
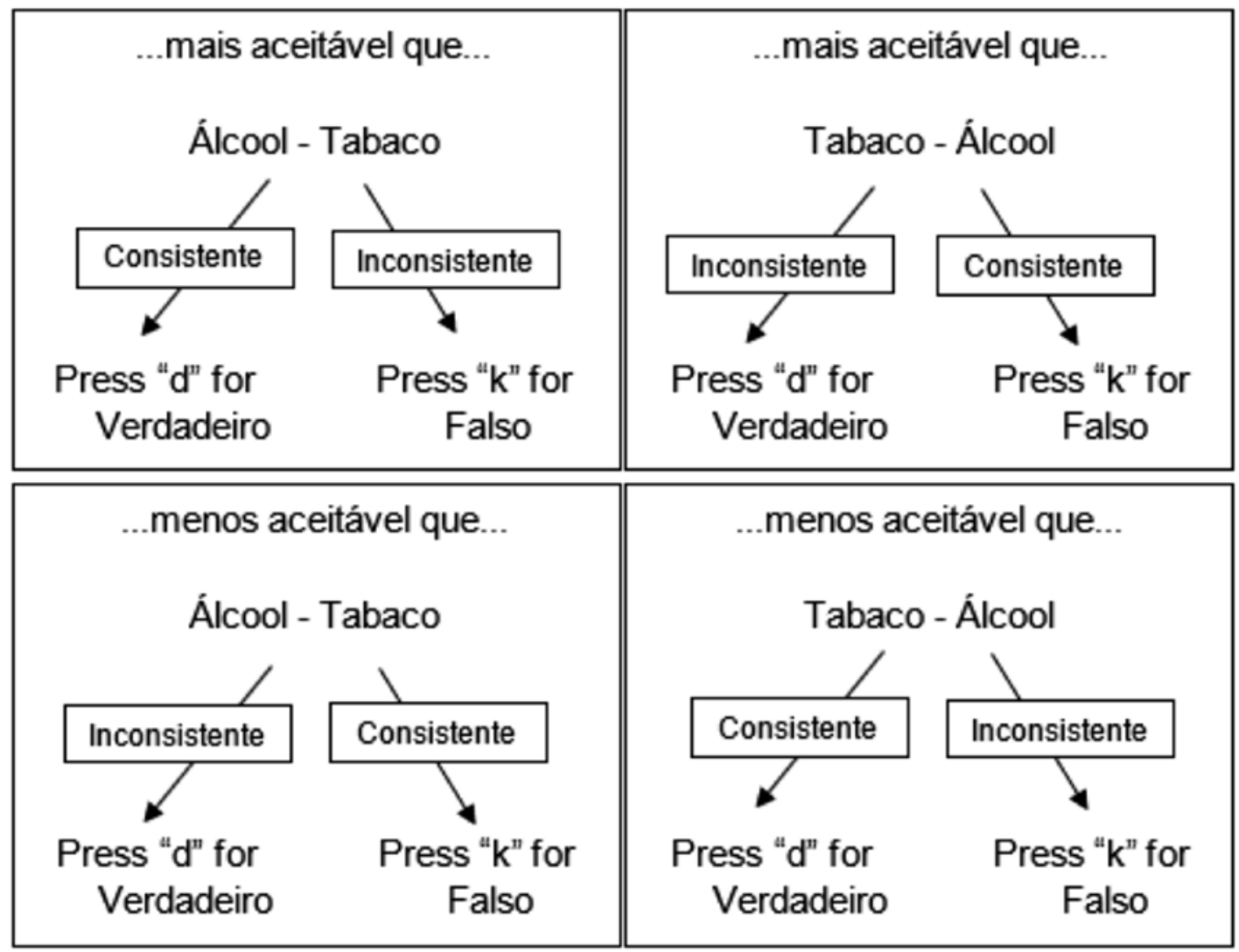

Figura 1. Exemplos de quatro tipos de tentativas IRAP. As setas e as caixas de texto indicam os padrões de respostas relacionais exigidos nos blocos consistentes e inconsistentes. 
mais aceitável que tabaco. As opções de respostas relacionais apresentadas em cada tentativa eram "verdadeiro" e "falso".

Essas tentativas eram agrupadas em blocos de 24 tentativas, nos quais se exigia que o participante ora emitisse respostas relacionais consistentes e ora inconsistentes com a ordem de aceitação baseada na prevalência de uso dessas substâncias. Assim, nos blocos consistentes, a regra era a seguinte: Responda às tentativas de forma a afirmar que (1) álcool é mais aceitável que tabaco, (2) tabaco é mais aceitável que maconha e (3) maconha é mais aceitável que cocaína. Esperava-se, portanto, que o participante respondesse "verdadeiro" nas tentativas em que o rótulo era "mais aceitável que" e os alvos eram álcool-tabaco, tabaco-maconha e maconha-cocaína. A opção "verdadeiro" também deveria ser escolhida nas tentativas em que o rótulo era "menos aceitável que" e os alvos eram cocaína-maconha, maconha-tabaco e tabaco-álcool. Ainda nos blocos consistentes, o participante deveria responder "falso" quando o rótulo era "mais aceitável que" e os alvos eram cocaína-maconha, maconha-tabaco e tabaco-álcool. Finalmente, a opção "falso" deveria ser escolhida também nas tentativas em que o rótulo era "menos aceitável que" e os alvos eram álcool-tabaco, tabaco-maconha e maconha-cocaína.

Nos blocos inconsistentes, o padrão de respostas relacionais a ser emitido pelo participante era invertido e a regra apresentava a seguinte instrução: Responda às tentativas de forma a afirmar que (1) cocaína é mais aceitável que maconha, (2) maconha é mais aceitável que tabaco e (3) tabaco é mais aceitável que álcool. A Figura 1 apresenta os padrões de respostas relacionais exigidos diante das diferentes combinações entre rótulo e alvo.

\section{Procedimento}

Após a assinatura do TCLE, cada participante preencheu o ASSIST (Henrique et al., 2004) e, em seguida, as escalas sobre o grau de aceitação para cada uma das substâncias psicoativas (i.e., álcool, tabaco, maconha e cocaína). Concluída esta etapa, os participantes foram conduzidos ao computador em que foi aplicado o IRAP.

Antes de iniciar a tarefa, era apresentada uma orientação geral sobre o procedimento. O participante era instruído a ler cuidadosamente o seguinte texto e esclarecer quaisquer dúvidas antes de dar prosseguimento à tarefa: "Olá! Nesta atividade, você irá visualizar duas palavras e uma expressão de comparação entre elas. Você precisa fazer a relação entre as palavras e a expressão, respondendo 'verdadeiro' ou 'falso', de acordo com a regra que lhe for dada pelo programa. A regra mudará ao longo do tempo. Para responder, você deverá pressionar a tecla ' $D$ ' para verdadeiro e ' $K$ ' para falso. Se você acertar, aparecerá uma nova tentativa. Se você errar, um ' $\mathrm{X}$ ' vermelho irá aparecer, portanto, responda novamente. O MAIS IMPORTANTE É RESPONDER O MAIS RÁPIDO POSSÍVEL E TENTAR NÃO COMETER ERROS! Coloque os dedos sobre as teclas "D" e 'K".

O participante era requisitado a acertar, no mínimo, $80 \%$ de respostas e a atingir uma latência média igual ou inferior a $4.000 \mathrm{~ms}$. Vale salientar que o valor da latência média foi definido com base em estudos pilotos cujos resultados não foram relatados aqui. De forma resumida, os valores $2.500 \mathrm{~ms}$, $3.000 \mathrm{~ms}$ e $3.500 \mathrm{~ms}$ foram testados. Em todos os casos, a grande maioria dos participantes falhou ao tentar obter os critérios de aprendizagem utilizando esses valores de latência média.

Em caso de não obtenção dos critérios, ele teria a possibilidade de repetir os blocos de treino até duas vezes. Ou seja, o participante era submetido a no mínimo dois e no máximo seis blocos, sempre alternados em pares consistentes e inconsistentes. Ao final dessa repetição, se o participante não alcançasse o critério, o procedimento era interrompido. Por outro lado, caso o critério fosse atingido, o participante era requisitado a fazer mais seis blocos. Para fins de comparação entre o desempenho nos blocos consistentes e inconsistentes, foram consideradas somente as latências registradas nos últimos seis blocos realizados.

\section{Resultados}

\section{Análise da medida explícita}

A Tabela 1 apresenta a média, a mediana e o desvio padrão dos escores nas escalas Likert calculados para cada uma das substâncias psicoativas a partir dos resultados obtidos com os 32 participantes. Segundo a estatística descritiva, o álcool foi 
a substância considerada mais aceitável, seguida pela maconha, tabaco e cocaína. Uma ANOVA de Friedman foi executada para comparação dos escores médios obtidos nas escalas de aceitação. Os resultados sugerem que as diferenças entre os escores obtidos nas quatro escalas foram estatisticamente significativas $(X 2=64,92, p<0,0001)$. Uma análise post hoc com o teste de Wilcoxon foi realizada com uma correção de Bonferroni, resultando em um nível de significância fixado em $p<0,008$. A análise post hoc revelou não ter encontrado diferenças entre os escores apenas para as escalas de aceitação de tabaco e maconha $(Z=-1,767, p=0,077)$. Em outras palavras, o tabaco foi qualificado de modo indistinto da maconha, em relação ao nível de aceitação.

\section{Tabela 1. Média, Mediana e Desvio Padrão dos Escores nas Escalas Likert.}

\begin{tabular}{llll}
\hline $\begin{array}{l}\text { Substância } \\
\text { Psicoativa }\end{array}$ & Média & Mediana & Desvio Padrão \\
\hline Álcool & 2,66 & 3 & 2,78 \\
Tabaco & $-0,13$ & -1 & 3,02 \\
Maconha & 1,06 & 2 & 3,22 \\
Cocaína & $-4,16$ & -4 & 2,08 \\
\hline
\end{tabular}

\section{Análise do tipo de tentativa por par de estímulos do IRAP}

Os dados brutos obtidos para cada participante foram transformados por meio de uma derivação do algoritmo $\mathrm{D}$ desenvolvido por Greenwald et al. (2003). O algoritmo D é tradicionalmente utilizado para controlar a variação individual na velocidade de resposta ocasionada pelas diferenças na habilidade cognitiva dos participantes, as quais podem atuar como uma variável confundidora quando forem analisadas diferenças entre grupos.

Os dados brutos foram transformados utilizando os seguintes passos:

1. Apenas as latências de resposta extraídas dos seis blocos finais foram utilizadas;

2. Dados dos participantes que apresentaram latência inferior a $300 \mathrm{~ms}$ em mais de $10 \%$ das tentativas foram desconsiderados para análise;
3. Tentativas com latências acima de $10.000 \mathrm{~ms}$ foram eliminadas da base de dados;

4. Foram calculados 36 desvios padrão para os quatro tipos de tentativa em cada um dos três pares de estímulos: quatro para as latências de resposta dos blocos de teste 1 e 2 , quatro para as latências de resposta dos blocos de teste 3 e 4 e mais quatro para as latências de resposta dos blocos de teste 5 e 6 , em cada um dos três pares de palavras.

5. Foram calculadas 72 médias de latências, uma para cada um dos quatro tipos de tentativa, em cada um dos seis blocos de teste, em cada um dos três pares de palavras;

6. Para cada par de bloco de teste, foi utilizado o passo 5 para calcular as pontuações de diferença para cada um dos quatro tipos de tentativa, subtraindo a latência média dos blocos consistentes, de cada tipo de tentativa, da latência média de dos blocos inconsistentes correspondentes, em cada um dos três pares de palavras; 7. Foram divididos cada escore de diferença obtido no passo 6 pelo seu desvio padrão correspondente no passo 4 , produzindo 36 pontuações $\mathrm{D}$, uma pontuação para cada tipo de tentativa, para cada um dos três pares de blocos de teste, para cada um dos três pares de palavras; 8. Foram calculadas 12 pontuações D para cada tipo de tentativa, em cada um dos três pares de palavras, calculando a média dos três escores $\mathrm{D}$ obtidos ao longo dos três pares de blocos de teste;

9. Foi obtido um escore composto D para cada um dos três pares de palavras, calculando a média dos quatro escores $\mathrm{D}$ obtidos por tipos de tentativa para cada par de palavra.

Desse modo, escores D-IRAP positivos significam que os participantes foram mais rápidos nos blocos consistentes em relação aos blocos inconsistentes, enquanto escores D-IRAP negativos representam o contrário. A Tabela 2 apresenta os escores D por tipo de tentativa por par de estímulos obtidos no passo 8 do algoritmo anteriormente apresentado.

Testes $t$ para os escores D-IRAP de cada tipo de tentativa por par de estímulos foram realizados para verificar se estes escores eram diferentes de 0 . 
Os resultados são estatisticamente significativos e diferentes de 0 apenas para tipo de tentativa "Mais aceitável X Álcool-Tabaco" [ $M=0,23, D P=0,54$, $t(31)=2,44, p=0,021]$ e para "Menos aceitável X Maconha-Tabaco" $[M=0,29, D P=0,61, t(31)=2,66$ $p=0,012]$. Neste sentido, analisando os pares de estímulos por tipos de tentativa, é possível dizer que os participantes apresentaram um viés no sentido de confirmar a relação em que álcool é mais aceitável que tabaco e maconha é menos aceitável que tabaco. Tabela 2. Média de Escore D-IRAP e Desvio Padrão
para Cada Tipo de Tentativa por Par de Estímulos.

\begin{tabular}{llll}
\hline Modelo & Estímulo alvo & M & DP \\
\hline Mais aceitável & Álcool - Tabaco & $0,23^{* *}$ & 0,54 \\
Mais aceitável & Tabaco - Álcool & 0,13 & 0,66 \\
Menos aceitável & Álcool - Tabaco & 0,09 & 0,59 \\
Menos aceitável & Tabaco - Álcool & 0,17 & 0,51 \\
\hline Mais aceitável & Tabaco - Maconha & 0,06 & 0,69 \\
Mais aceitável & Maconha - Tabaco & $-0,12$ & 0,62 \\
Menos aceitável & Tabaco - Maconha & 0,13 & 0,63 \\
Menos aceitável & Maconha - Tabaco & $0,29 * *$ & 0,61 \\
\hline Mais aceitável & Maconha - Cocaína & 0,01 & 0,70 \\
Mais aceitável & Cocaína - Maconha & $-0,06$ & 0,49 \\
Menos aceitável & Maconha - Cocaína & $-0,01$ & 0,67 \\
Menos aceitável & Cocaína - Maconha & $-0,25$ & 0,70 \\
\hline ** p<0,05 & & &
\end{tabular}

\section{Análise dos pares de palavras do IRAP}

A Tabela 3 apresenta escore D-IRAP para cada um dos pares de palavras (ver passo 9). Somente para o par álcool-tabaco foi possível obter um escore estatisticamente significativo e diferente de 0 [álcool-tabaco: $M=0,16, D P=0,33, t(31)=2,66, p=0,012$; tabaco-maconha: $M=0,09, D P=0,38, t(31)=1,35$, $p=0,188$; maconha-cocaína: $M=-0,07, D P=0,41$, $t(31)=-1,10, p=0,282]$. Os resultados sugerem que os participantes globalmente qualificam álcool como mais aceitável que tabaco, mas não é possível afirmar que exista um viés de aceitação para tabaco comparado a maconha, nem maconha comparado a cocaína.
Tabela 3. Média de Escore D-IRAP e Desvio Padrão para Cada Par de Palavras.

\begin{tabular}{lll}
\hline Estímulo alvo & $\mathrm{M}$ & $\mathrm{DP}$ \\
\hline Álcool - Tabaco & $0,16^{\star \star}$ & 0,33 \\
Tabaco - Maconha & 0,09 & 0,38 \\
Maconha - Cocaína & $-0,07$ & 0,41 \\
\hline${ }^{\star \star} p<0,05$ & & \\
\hline
\end{tabular}

\section{Experiência prévia com o uso de álcool}

Como descrito anteriormente, todos os participantes do presente experimento afirmaram não possuir qualquer tipo de experiência com o uso de tabaco, maconha ou cocaína, mas parte deles admitiu ter feito uso de álcool. Por esse motivo, a Tabela 4 apresenta uma análise post hoc dos resultados do IRAP em que os 32 participantes foram divididos entre aqueles que não tinham experiência com o uso de álcool $(n=9)$ e aqueles que já fizeram uso dessa substância $(\mathrm{n}=23)$.

\section{Tabela 4. Média de Escore D-IRAP e Desvio Padrão Para Cada Grupo e Par de Estímulos Alvos.}

\begin{tabular}{llll}
\hline Grupo & Estímulo alvo & M & DP \\
\hline Sem experiência & Álcool - Tabaco & $-0,02$ & 0,31 \\
& Tabaco - Maconha & 0,07 & 0,35 \\
& Maconha - Cocaína & $-0,13$ & 0,43 \\
\hline \multirow{2}{*}{ Com experiência } & Álcool - Tabaco & $0,23^{\star *}$ & 0,32 \\
& Tabaco - Maconha & 0,10 & 0,40 \\
& Maconha - Cocaína & $-0,06$ & 0,41 \\
\hline${ }^{* *} p<0,05$ & & & \\
\end{tabular}

O teste $t$ para os escores D-IRAP obtidos sugere que os resultados foram diferentes de 0 para o par álcool-tabaco no grupo com experiência $[M=0,23$, $D P=0,32, t(22)=3,36, p=0,003]$, mas não no grupo sem experiência $[M=-0,02, D P=0,31, t(8)=-0,21$, $p=0,84]$. Por meio de uma ANOVA unidirecional, verificou-se que a diferença entre os grupos pode ter ocorrido em razão de erro amostral, apesar do nível de significância para rejeição da hipótese nula ter ficado muito próximo de $0,05[F(1,30)=3,91$, $p=0,057]$. Embora as diferenças nas médias dos grupos sejam marginalmente significativas, estes 
dados sugerem que, para o grupo sem experiência prévia com o uso de álcool, nenhuma substância é mais aceitável comparada à outra, enquanto para o grupo com experiência, álcool é mais aceitável que tabaco e, em teoria, mais aceitável que as demais substâncias. De acordo com o IRAP, portanto, é possível sugerir que a aceitação do álcool em relação ao tabaco e às demais drogas esteve relacionada à história prévia de consumo de bebidas alcoólicas.

\section{Discussão}

O objetivo do presente estudo era avaliar a existência de um responder relacional de comparação com base na aceitação de substâncias psicoativas em pessoas com baixos índices de consumo de álcool e sem história de uso de tabaco, maconha e cocaína. Esperava-se que essa ordem de aceitação fosse semelhante à ordem de consumo dessas mesmas substâncias no Brasil. Os resultados encontrados tanto na medida explícita quanto na implícita, no entanto, refutaram essa hipótese. De acordo com os resultados obtidos na medida explícita, o álcool foi considerado mais aceitável que tabaco e maconha, substâncias que não foram significativamente diferentes entre si em relação ao grau de aceitação. A cocaína, por sua vez, foi considerada a menos aceitável dentre as substâncias avaliadas. Assim, tabaco e maconha foram indistintamente aceitáveis, contrariando a hipótese que tabaco seria qualificado com maior grau de aceitação em relação a maconha. Os resultados observados no IRAP, por sua vez, sugerem uma relação de maior aceitação de álcool comparado a tabaco e de indiferenciação em relação a aceitação de tabaco comparado a maconha e maconha comparada a cocaína. Desse modo, é possível afirmar apenas que o álcool foi mais aceitável quando comparado as demais substâncias.

As diferenças entre os resultados obtidos por meio de medidas explícitas e implícitas podem ser explicadas pelo modelo REC (Relational Elaboration and Coherence; Barnes-Holmes, Barnes-Holmes et al., 2010; Cullen et al., 2009). Segundo este modelo, as respostas relacionais se desdobram ao longo do tempo, havendo aquelas que são emitidas relativamente rápido e outras que são emitidas em uma janela de tempo maior.
As primeiras são chamadas de respostas relacionais breves e imediatas (BIRR - brief and immediate relacional response), enquanto as últimas são chamadas de respostas relacionais estendidas e elaboradas (EERR - extended and elaborated relacional response). Assim sendo, ambas as respostas, BIRRs e EERRs, são da mesma natureza, mas diferem quanto ao parâmetro temporal, sendo acessadas por procedimentos distintos. Uma tarefa que exija rapidez e precisão (i.e., IRAP) provavelmente avaliará BIRRs, enquanto tarefas que garantam a emissão de uma resposta sem limite de tempo (i.e., escalas Likert) poderão avaliar EERRs. Mais recentemente, ao proporem o modelo Multidimensional, Multiníveis (Barnes-Holmes, Barnes-Holmes et al., 2017; Barnes-Holmes, Finn et al., 2017), BarnesHolmes e colaboradores argumentaram que as respostas relacionais devem ser analisadas de acordo com os níveis de derivação e complexidade relacional. O EERR, então, pode ser compreendido como um tipo de resposta que recruta coerência entre várias redes de relações, apresentando altos níveis de complexidade e derivação, diferentemente do BIRR, que tipicamente apresenta baixos níveis de complexidade e derivação.

No estudo de Power et al. (2009), por exemplo, as respostas sobre preferência étnica na medida explícita pareciam controladas pelas relações com altos níveis de complexidade, coerentes com o que seria "politicamente correto" (e.g., julgar africanos como mais simpáticos que americanos em razão de não querer parecer favorável a um povo que se acredita explorar o terceiro mundo) enquanto o padrão de respostas exibido no IRAP parecia controlado por relações menos complexas como a similaridade social percebida entre as etnias. No presente estudo, ainda que os participantes não considerem a cocaína como sendo uma substância aceitável, podem ter sido mais rigorosos na medida explícita em razão de isso ser coerente com a rede relacional mais extensa e reforçada pela comunidade verbal, em que esta substância é considerada como sendo potencialmente mais perigosa que as demais para o indivíduo e para a sociedade. Seguindo a mesma linha, os participantes sem experiência prévia com álcool, embora inicialmente não qualificassem qualquer substância como mais aceitável em relação a outra, podem ter emitido uma resposta 
de maior aceitação de álcool em função de isso ser mais coerente com a rede relacional mais complexa que qualifica essa substância como positiva e desejável socialmente.

A utilização de uma latência máxima de 4.000 ms no presente experimento merece ser justifica$\mathrm{da}$, pois esse valor talvez dificultasse o acesso aos BIRRs - uma vez que se trata de um valor consideravelmente maior do que os $2.000 \mathrm{~ms}$ geralmente utilizados em estudos com o IRAP (e.g., Carpenter et al., 2012; Drake et al., 2015; Leech et al., 2016). Barnes-Holmes, Murphy et al. (2010) demonstraram que a utilização de $2.000 \mathrm{~ms}$ como critério de latência máxima parece aumentar o tamanho do efeito IRAP e a confiabilidade interna quando comparado a utilização de $3.000 \mathrm{~ms}$. Contudo, o valor de $4.000 \mathrm{~ms}$ foi determinado a partir da realização de estudos pilotos com valores que variaram entre 2.500 até $3.500 \mathrm{~ms}$, sendo que a grande maioria dos participantes falhava em alcançar os critérios estabelecidos nessas condições. Mesmo utilizando o valor de $4.000 \mathrm{~ms}$, metade dos participantes deste estudo não conseguiu atingir os critérios estabelecidos. É reconhecida a limitação em relação à literatura uma vez que a utilização de valores menores como critério de latência poderia tornar mais claro e robusto o efeito observado. Entretanto, nossos dados não parecem estar comprometidos enquanto uma medida implícita, pois nesse mesmo artigo, Barnes-Holmes, Murphy et al. também argumentam que o valor de $2.000 \mathrm{~ms}$ pode ser curto demais para alguns tipos de estímulos ou populações submetidas ao IRAP. No caso do presente experimento, a utilização de frases como rótulo e pares de palavras como alvo certamente se constitui em elemento que deveria ocasionar um aumento no limiar de tempo necessário para que os participantes emitissem as respostas relacionais.

Ademais, os resultados apresentados na Tabela 4 sugerem que a experiência prévia com o consumo de álcool modulou os resultados obtidos no IRAP. A sensibilidade do IRAP à variação do comportamento aberto foi demonstrada em vários estudos anteriores, sendo o efeito IRAP capaz de distinguir vegetarianos de comedores de carne (BarnesHolmes, Murtagh et al., 2010), pacientes com anorexia nervosa de não clínicos (Parling et al., 2012), indivíduos disfóricos de não disfóricos (Remue et al., 2013), pacientes psiquiátricos com ideação suicida de não clínicos (Hussey et al., 2016), entre outros. Especificamente no domínio das substâncias psicoativas, estudos anteriores também demonstraram a capacidade do IRAP em diferenciar grupos de não usuários de usuários de substâncias, bem como diferenciar participantes bem-sucedidos daqueles mal-sucedidos no tratamento para dependência de cocaína (Cagney et al., 2017; Carpenter et al., 2012; Vahey et al., 2010). Cagney et al. (2017), por exemplo, demonstraram que o IRAP foi capaz de diferenciar grupos de adolescentes e adultos fumantes de não fumantes. $\mathrm{O}$ procedimento IRAP basicamente consistia em relacionar os rótulos "fumantes" e "não fumantes" ora a alvos com atributos positivos (e.g., legal, popular, divertido) ora com atributos negativos (e.g., chato, fraco, perdedor). Foram utilizadas duas medidas explícitas: escalas Likerts e o "termômetro de sentimentos" (Feeling Thermomether). Os resultados do IRAP mostraram que os participantes não fumantes apresentaram um viés global neutro (i.e., um escore D-IRAP geral próximo de 0 ), enquanto os participantes fumantes apresentaram um viés global pró-fumantes. Nas medidas explícitas, os resultados mostraram que, mesmo sendo significativas as diferenças entre os fumantes e não fumantes, todos os participantes apresentaram um viés pró-não fumantes. Em resumo, além de ter apresentado diferenças entre as medidas explícitas e implícitas acessando a temática do uso de substâncias, o estudo de Cagney et al. demonstrou como o IRAP conseguiu ser sensível às diferenças dos comportamentos abertos exibidos entre fumantes e não fumantes por meio da avaliação das atitudes implícitas de modo semelhante ao apresentado na análise post hoc do presente estudo. Neste sentido, estudos futuros poderiam selecionar participantes com e sem experiência no uso das demais substâncias, assim como controlar a quantidade de participantes em cada grupo a fim de verificar especificamente se o padrão de aceitação encontrado no IRAP para os participantes com experiência no uso álcool se reproduz em usuários das demais drogas, ou seja, qualificar como mais aceitáveis as substâncias consumidas por eles e igualmente aceitáveis aquelas não consumidas.

Ainda relacionado ao consumo de substâncias, vale salientar que as informações referentes a 
esse tópico foram obtidas por meio de autorrelato. Assim sendo, apesar da garantia de confidencialidade, não há como excluir a possibilidade que os participantes tenham optado por não admitir o uso de algumas dessas substâncias, principalmente daquelas que são ilícitas. Assim sendo, futuros experimentos que desejarem investiguem se a experiência prévia com o consumo de determinada substância pode modular os resultados obtidos no IRAP devem pensar em formas mais confiáveis de se obter esses dados.

Adicionalmente, os resultados obtidos no presente experimento também podem estar relacionados a alguns aspectos inerentes a procedimentos destinados a investigar a existência de ordem relacionais. O primeiro aspecto a ser destacado está relacionado à necessidade de se utilizar pares de palavras como alvo; por exemplo, ao pensar na disposição espacial dos pares de palavras, (1) o termo "tabaco" era apresentado à esquerda em duas combinações diferentes (i.e., tabaco-álcool e tabaco-maconha), enquanto (2) o termo "álcool" era apresentado nessa mesma posição em apenas uma combinação (i.e., álcool-tabaco). Os resultados mostraram que as latências brutas foram significativamente menores nas tentativas do tipo 2 quando comparadas às tentativas do tipo 1 . Por haver uma única possibilidade de combinação, ao ler a palavra "álcool", tornava-se possível inferir que a segunda palavra era "tabaco", diminuindo a latência nessas tentativas. Neste sentido, pode ser necessário estabelecer procedimentos para garantir que os alvos sejam lidos por completo antes da emissão da resposta relacional. Eventualmente, a implementação de um procedimento de read-aloud como realizado no experimento 2 de Finn et al. (2018) poderia diminuir tais efeitos.

Além disso, a conversão dos estímulos em frases completas poderia, se não eliminar este efeito da leitura parcial, ao menos minimizá-lo. Aparentemente, transformar um IRAP típico em um IRAP de Língua Natural (Natural Language IRAP) não repercute em diferenças significativas, embora possa encorajar os participantes a responderem ao rótulo ou alvo da maneira como foi programado pelo experimentador (Kavanagh et al., 2016). Adicionalmente, a conversão em frases também poderia resolver uma possível limitação do presente experimento com relação aos termos apresentados como rótulos e alvos, a saber, o fato de não ser possível identificar com clareza se os participantes estavam considerando a aceitação em um nível individual ou em um nível social ao emitirem suas respostas. Não por acaso, durante o preenchimento da escala explícita, alguns participantes perguntavam ao pesquisador se deveriam marcar o quanto as substâncias eram aceitáveis para ele ou para os outros. A utilização de frases como 'Em minha opinião, o uso da maconha é mais aceitável do que do tabaco' esclareceria qual a moldura dêitica deveria ser considerada na tarefa.

Outro aspecto inerente aos experimentos sobre a existência de ordens relacionais refere-se ao uso de frases na forma negativa. Os dados coletados do IRAP sugeriram que os participantes responderam mais lentamente aos rótulos "menos aceitável que" em relação ao "mais aceitável que". Provavelmente, esse resultado está relacionado ao fato de sermos expostos de forma mais frequente a descrições na forma positiva que na forma negativa (e.g., mais bonito ao invés de menos bonito) (O'Shea et al., 2016). Por exemplo, na tentativa "tabaco menos aceitável que álcool”, o participante privadamente pode ter emitido uma resposta relacional de oposição em que "menos" é o contrário de "mais". Então, uma segunda resposta relacional seria necessária para especificar que para a frase "tabaco mais aceitável que álcool" a resposta correta é "verdadeiro". Assim sendo, para "tabaco menos aceitável que álcool", que é o oposto da frase anterior, a resposta correta será "falso". Essa exigência por respostas mais complexas implica em diferenças nas latências não relacionadas ao objetivo principal do experimento. Além disso, a complexidade das redes de relações necessárias para a emissão das respostas relacionais pode também explicar, ao menos em parte, a necessidade de se utilizar um valor de latência média elevado se comparado aos valores usualmente empregados em tarefas de IRAP.

Neste ponto, parece-nos importante salientar que, com base na revisão feita para a escrita desse trabalho, os experimentos publicados por Power et al. (2009) parecem ter sido os únicos a investigar a existência de ordens relacionais por meio do IRAP. Assim, talvez seja necessário a formulação de experimentos que testem as propriedades do 
procedimento IRAP com relação ao responder relacional de comparação em questões mais básicas como, por exemplo, uma tarefa com rótulos como "menor que" e "maior que" (uma propriedade física) e alvos como rato, cachorro, leão e elefante. Assim sendo, as ordens avaliadas seriam rato menor que cachorro, cachorro menor que leão e leão menor que elefante. Resultados advindos de um experimento como esse poderiam ajudar a esclarecer quanto os dados obtidos por meio do IRAP são influenciados pelas limitações do procedimento apontadas anteriormente (i.e., apresentação de pares de palavras como estímulos alvo e frases em sua forma negativa).

Por fim, os níveis de desaprovação relacionados a uma determinada substância psicoativa são tidos como um fator de proteção para o seu uso futuro (Bachman et al., 1990), ou seja, quanto maior o nível de desaprovação de uma substância, menor seria a probabilidade de uma pessoa fazer uso dela ao longo da vida. No entanto, a relação causal entre as atitudes relacionadas a substâncias psicoativas e o comportamento de uso ainda é um assunto controverso, com alguns estudos sugerindo que atitudes precedem o uso enquanto outros sugerem o contrário (de Leeuw et al., 2008; Jones \& Gordon, 2017; van der Zwaluw et al., 2013). Salas-Wright et al. (2015), por exemplo, apresentaram resultados que contrariaram a relação esperada entre a redução na desaprovação e o aumento da prevalência de uso. Os autores examinaram as tendências em desaprovação e o uso de maconha entre adolescentes e adultos jovens nos Estados Unidos no período de 2002 a 2013. Os resultados mostraram que a proporção de adultos jovens que reportavam uma forte desaprovação ao uso de maconha decresceu de 40,5\% em 2002 para 22,6\% em 2013. Por outro lado, o aumento na prevalência de consumo no último ano não foi proporcional à redução da desaprovação (i.e., de 29,7\% em 2002 para 31,9\% em 2013).

Até a presente data, grande parte dos estudos transversais ou longitudinais com grandes amostras que avaliaram atitudes sobre substâncias psicoativas o fizeram utilizando medidas explícitas, assim como Salas-Wrigth et al. (2015). Utilizando tais medidas, é possível que ocorram distorções semelhantes à evidenciada pelo estudo de Cagney et al. (2017) em que fumantes exibiram um viés pró-fumantes na medida implícita e o contrário na medida explícita. No estudo de Salas-Wright et al., por exemplo, é possível que, caso fossem empregadas medidas implícitas para avaliar as atitudes, a mudança na prevalência de uso ao longo da década correspondesse melhor às mudanças nas atitudes, o que eventualmente deixaria claro que a redução da desaprovação da maconha apresentada pelo estudo é reflexo da diferença entre os tipos de responder relacionais avaliados por meio de medidas explícitas e implícitas. Desse modo, futuros estudos que intencionem avaliar a relação causal entre atitudes sobre substâncias psicoativas e seu uso poderiam se beneficiar do emprego de medidas implícitas para este fim.

\section{Referências}

Bachman, J. G., Johnston, L. D., \& O’Malley, P. M. (1990). Explaining the recent decline in cocaine use among young adults: Further evidence that perceived risks and disapproval lead to reduced drug use. Journal of Health and Social Behavior, 31, 173-184.

Barnes-Holmes, D., Barnes-Holmes, Y., Luciano, C., \& McEnterggart, C. (2017). From the IRAP and REC model to a multi-dimensional multi-level framework for analysing the dynamics of arbitrarily applicable relational responding. Journal of Contextual Behavior Science, 6, 434445. https://doi.org/10.1016/j.jcbs.2017.08.001

Barnes-Holmes, D., Barnes-Holmes, Y., Stewart, I., \& Boles, S. (2010). A sketch of the Implicit Relational Assessment Procedure (IRAP) and the Relational Elaboration and Coherence (REC) model. The Psychological Record, 60, 527-542. https://doi.org/10.1007/BF03395726

Barnes-Holmes, D., Finn, M., McEnterggart, C., \& Barnes-Holmes, Y. (2017). Derived stimulus relations and their role in a behaviour-analytic account of human language and cognition. The Behavior analyst, 41, 155-173. https://doi. org/10.1007/s40614-017-0124-7

Barnes-Holmes, D., Hayden, E., \& Barnes-Holmes, Y. (2008). The Implicit Relational Assessment Procedure (IRAP) as a response-time and event-related-potentials methodology for tes- 
ting natural verbal relations: A preliminary study. The Psychological Record, 58, 497-516. https://doi.org/10.1007/BF03395634

Barnes-Holmes, D., Murphy, A., Barnes-Holmes, Y., \& Stewart, I. (2010). The Implicit Relational Assessment Procedure: Exploring the impact of private versus public contexts and the response latency criterion on pro-white and anti-black stereotyping among white Irish individuals. The Psychological Record, 60, 57-79. https://doi. org/10.1007/BF03395694

Barnes-Holmes, D., Murtagh, L., \& Barnes-Holmes, Y. (2010). Using the Implicit Association Test and the Implicit Relational Assessment Procedure to measure attitudes toward meat and vegetables in vegetarians and meat-eaters. The Psychological Record, 60, 287-306. https:// doi.org/10.1007/BF03395708

BRASIL. (2012). II Levantamento nacional sobre o uso de álcool, tabaco e outras drogas entre universitários das 27 capitais brasileiras. Secretaria Nacional de Políticas sobre Drogas; GREA/ IPQ-HCFMUSP. Brasília: SENAD.

Cagney, S., Harte, C., Barnes-Holmes, D., BarnesHolmes, Y., \& McEnteggart, C. (2017). Response biases on the IRAP for adults and adolescents with respect to smokers and nonsmokers: The impact of parental smoking status. The Psychological Record, 67, 473-483. https:// doi.org/10.1007/s40732-017-0249-9

Carpenter, K. M., Martinez, D., Vadhan, N. P., Barnes-Holmes, D., \& Nunes, E. V. (2012). Measures of attentional bias and relational responding are associated with behavioral treatment outcome for cocaine dependence. The American Journal of Drug and Alcohol Abuse, 38, 146-154. https://doi.org/10.3109/00952990 .2011 .643986

Cullen, C., Barnes-Holmes, D., Barnes-Holmes, Y., \& Stewart, I. (2009). The Implicit Relational Assessment Procedure (IRAP) and the malleability of ageist attitudes. The Psychological Record, 59, 591-620. https://doi.org/10.1007/ BF03395683

Dawson, D. L., Barnes-Holmes, D., Gresswell, D. M., Hart, A. J., \& Gore, N. J. (2009). Assessing the implicit beliefs of sexual offenders using the implicit relational assessment procedure: A first study. Sexual Abuse, 2, 57-75. https://doi. org/10.1177/1079063208326928

de Leeuw, R. N. H., Engels, R. C. M. E., Vermulst, A. A., \& Scholte, R. H. J. (2008). Do smoking attitudes predict behaviour? A longitudinal study on the bi-directional relations between adolescents' smoking attitudes and behaviours. Addiction, 103, 1713-1721. https://doi. org/10.1111/j.1360-0443.2008.02293.x

de Rose, J. C., \& Bortoloti, R. (2007). A equivalência de estímulos como modelo do significado. Acta Comportamentalia, 15, 83-102.

Drake, C. E., Kramer, S., Habib, R., Schuler, K., Blankenship, L., \& Locke, J. (2015). Honest politics: Evaluating candidate perceptions for the 2012 US election with the Implicit Relational Assessment Procedure. Journal of Contextual Behavioral Science, 4, 129-138. http://doi. org/10.1016/j.jcbs.2015.04.004

Farrell, L., \& McHugh, L. (2017). Examining gender-STEM bias among STEM and non-STEM students using the Implicit Relational Assessment Procedure (IRAP). Journal of Contextual Behavioral Science, 6, 80-90. https:// doi.org/10.1016/j.jcbs.2017.02.001

Finn, M., Barnes-Holmes, D., \& McEnterggart, C. (2018). Exploring the single-trial-type-dominance-effect in the IRAP: developing a Differential Arbitrarily Applicable Relational Responding Effects (DAARRE) model. The Psychological Record, 68, 11-25. https://doi. org/10.1007/s40732-017-0262-z

Greenwald, A. G., Nosek, B. A., \& Banaji, M. R. (2003). Understanding and using the Implicit Association Test: I. An improved scoring algorithm. Journal of Personality and Social Psychology, 85, 197-216. https://doi. org/10.1037/0022-3514.85.2.197

Henrique, I. F. S., Micheli, D., Lacerda, R. B., Lacerda, L. A., \& Formigoni, M. L. O. S. (2004). Validação da versão brasileira do teste de triagem do envolvimento com álcool, cigarro e outras substâncias (ASSIST). Revista da Associação Médica Brasileira, 50, 199-206.

Hughes, S., Barnes-Holmes, D., \& De Houwer, J. (2011). The Dominance of Associative theorizing in implicit attitude research: propositional and behavioral alternatives. The Psychological 
Record, 61, 465-496. https://doi.org/10.1007/ BF03395772

Hussey, I., Barnes-Holmes, D., \& Booth, R. (2016). Individuals with current suicidal ideation demonstrate implicit "fearlessness of death." Journal of Behavior Therapy and Experimental Psychiatry, 51, 1-9. https://doi.org/10.1016/j.jbtep.2015.11.003

Jones, S. C., \& Gordon, C. S. (2017). A systematic review of children's alcohol-related knowledge, attitudes and expectancies. Preventive Medicine, 105, 19-31. https://doi.org/10.1016/j. ypmed.2017.08.005

Kavanagh, D., Hussey, I., McEnteggart, C., BarnesHolmes, Y., \& Barnes-Holmes, D. (2016). Using the IRAP to explore natural language statements. Journal of Contextual Behavioral Science, 5, 247-251. https://doi.org/10.1016/j. jcbs.2016.10.001

Kiepper, A., \& Esher, A. (2014). A regulação da maconha no Senado Federal: Uma pauta da saúde pública no Brasil. Caderno de Saúde Pública, 30 (8), 1-3. http://dx.doi.org/10.1590/0102$-311 X P E 01081$

Laranjeira, R. (2010). Legalização de drogas e a saúde pública. Ciência \& Saúde Coletiva, 15, 621-631. http://dx.doi.org/10.1590/S141381232010000300002

Laranjeira, R., Madruga, C. S., Ribeiro, M., Pinsky, I., Caetano, R., \& Mitsuhiro, S. S. (2012). II Levantamento Nacional de Álcool e Drogas (LENAD) - 2012. Instituto Nacional de Ciência e Tecnologia para Políticas Públicas de Álcool e Outras Drogas (INPAD), São Paulo: UNIFESP.

Leech, A., Barnes-Holmes, D., \& Madden, L. (2016). The implicit relational assessment procedure (IRAP) as a measure of spider fear, avoidance, and approach. The Psychological Record, 66, 337-349. https://doi.org/10.1007/s40732016-0176-1

Levin, M. E., Hayes, S. C., \& Waltz, T. (2010). Creating an implicit measure of cognition more suited to applied research: A test of the Mixed Trial-Implicit Relational Assessment Procedure (MT-IRAP). International Journal of Behavioral Consultation and Therapy, 6, 245-
262. http://dx.doi.org/10.1037/h0100911.

MacCoun, R. J. (1993). Drugs and the law: A psychological analysis of drug prohibition. Psychological Bulletin, 113, 497-512. https://doi. org/10.1037/0033-2909.113.3.497

Mizael, T. M., dos Santos, S. L., \& de Rose, J. C. (2016). Contribuições do paradigma de equivalência de estímulos para o estudo das atitudes. Interação em Psicologia, 20, 124-134. http:// dx.doi.org/10.5380/psi.v20i2.46278

Nutt, D., King, L., \& Phillips, L. (2010). Drug harms in the UK: A multicriteria decision analysis. The Lancet, 376, 1558-1565. https://doi. org/10.1016/S0140-6736(10)61462-6

O'Shea, B., Watson, D. G., \& Brown, G. D. A. (2016). Measuring implicit attitudes: a positive framing bias flaw in the Implicit Relational Assessment Procedure (IRAP). Psychological Assessment, 28, 158-170. https://doi.org/10.1037/pas0000172

Parling, T., Cernvall, M., Stewart, I., BarnesHolmes, D., \& Ghaderi, A. (2012). Using the Implicit Relational Assessment Procedure to compare implicit pro-thin/anti-fat attitudes of patients with anorexia nervosa and non-clinical controls. Eating Disorders, 20, 127-143. https:// doi.org/10.1080/10640266.2012.654056

Power, P., Barnes-Holmes D., Barnes-Holmes, Y., \& Stewart, I. (2009). The Implicit Relational Assessment Procedure (IRAP) as a measure of implicit relative preferences: A first study. The Psychological Record, 59, 621-640. https://doi. org/10.1007/BF03395684

Remue, J., de Houwer, J., Barnes-Holmes, D., Vanderhasselt, A., \& de Raedt, R. (2013). Selfesteem revisited: Performance on the implicit relational assessment procedure as a measure of self- versus ideal self-related cognitions in dysphoria. Cognition \& Emotion, 27, 1441-1449. https://doi.org/10.1080/02699931.2013.786681

Salas-Wright, C. P., Vaughn, M. G., Todic, J., Córdova, D., \& Perron, B. E. (2015). Trends in the disapproval and use of marijuana among adolescents and young adults in the United States: 2002-2013. The American Journal of Drug and Alcohol Abuse, 41, 392-404. https:// doi.org/10.3109/00952990.2015.1049493

Skinner, B. F. (1953). Science and human behavior. Macmillan. 
Vahey, N. A., Boles, S. \& Barnes-Holmes, D. (2010). Measuring adolescents' smoking-related social identity preferences with the Implicit Relational Assessment Procedure (IRAP) for the first time: A starting point that explains later IRAP evolutions. International Journal of Psychology and Psychological Therapy, 10, 453-474.

van der Zwaluw, C. S., Kleinjan, M., Lemmers, L., Spijkerman, R., \& Engels, R. C. M. E. (2013). Longitudinal associations between attitudes towards binge drinking and alcohol-free drinks, and binge drinking behavior in adolescence. Addictive Behaviors, 38, 2110-2114. https://doi. org/10.1016/j.addbeh.2013.01.012

Wilkinson, S. T., Yarnell, S., Radhakrishnan, R., Ball, S. A., \& D’Souza, D. C. (2016). Marijuana legalization: Impact on physicians and public health. Annual Review of Medicine, 67, 453-466. https://doi.org/10.1146/annurev-med-050214-013454 